\title{
A novel ex vivo porcine model of acid-induced esophageal damage for preliminary functional evaluations of anti-gastroesophageal reflux disease medical devices
}

\author{
Domenico Ventrella ${ }^{1}$ (i), Roberta Salaroli ${ }^{1}$, Alberto Elmi ${ }^{1}$ (D), Giacomo Carnevali ${ }^{1}$, Monica Forni ${ }^{1}$ (D), Fabio Baldi ${ }^{2,3}$ (D) and \\ Maria Laura Bacci ${ }^{1}$ (i)
}

1. Department of Veterinary Medical Sciences, University of Bologna, Ozzano dell'Emilia (BO), Italy; 2. Center for the Study of Diseases of the Esophagus, University of Bologna, Bologna, Italy; 3. GVM Care and Research, Cotignola (RA), Italy.

Corresponding author: Alberto Elmi, e-mail: alberto.elmi2@unibo.it

Co-authors: DV: domenico.ventrella2@unibo.it, RS: roberta.salaroli@unibo.it, GC: g.carnevali@anas.it, MF: monica.forni@unibo.it, FB: fa.baldi@libero.it, MLB: marialaura.bacci@unibo.it

Received: 20-07-2020, Accepted: 04-11-2020, Published online: 21-12-2020

doi: www.doi.org/10.14202/vetworld.2020.2728-2735 How to cite this article: Ventrella $D$, Salaroli $R$, Elmi $A$, Carnevali G, Forni M, Baldi F, Bacci ML (2020) A novel ex vivo porcine model of acid-induced esophageal damage for preliminary functional evaluations of anti-gastroesophageal reflux disease medical devices, Veterinary World, 13(12): 2728-2735.

\begin{abstract}
Aim: The aim of the study was to set up a porcine ex vivo model of acid-induced damage and to evaluate its performance by means of multichannel intraluminal impedance and $\mathrm{pH}(\mathrm{MII}-\mathrm{pH})$ live recording, histology, and Evans blue (EB) permeability assay.

Materials and Methods: Thirteen esophagi, collected at a slaughterhouse, were ablated of their sphincters, pinned upright on a support, and placed in a thermostatic hood at $37^{\circ} \mathrm{C}$ with two infusion tubes and an MII-pH probe inserted in the top end. Three esophagi (histology controls) were only left in the hood for $3.5 \mathrm{~h}$ before sampling, while the remaining organs underwent the experimental protocol including saline infusion and recovery recording, and acid solution infusion and recovery recording.

Results: MII-pH analysis highlighted a significantly stronger decrease during acid infusion when compared to saline, but a better post-infusion recovery for saline solution. At the end of the protocol, MII was still statistically lower than baseline. The acid-damaged esophagi significantly absorbed more EB dye, and histology revealed strong mucosal exfoliation.

Conclusion: The proposed model of esophageal acid damage seems to be repeatable, reliable, and achievable using organs collected at the slaughterhouse. MII recording proved to have good sensitivity in detecting mucosal alterations also in ex vivo trials.
\end{abstract}

Keywords: esophagus ex vivo model, Evans blue permeability assay, gastroesophageal reflux disease, multichannel intraluminal impedance, pig.

\section{Introduction}

Gastroesophageal reflux is a physiological event occurring during and after meals that becomes pathological, under the name of gastroesophageal reflux disease (GERD), when the reflux of stomach contents causes symptoms and complications [1]. Common GERD-associated symptoms are heartburn and regurgitation [2] while, in worst cases, other "extra-esophageal symptoms" such as chronic cough, aspiration pneumonia, pulmonary fibrosis, hoarseness, and dental erosions are reported [3]. The disease shows an estimated prevalence of $20-25 \%$ in industrialized countries, with an increasing rate mainly due to the diffusion of obesity [4], and has high economic impact, with both direct and indirect costs

Copyright: Ventrella, et al. Open Access. This article is distributed under the terms of the Creative Commons Attribution 4.0 International License (http://creativecommons.org/licenses/ by/4.0/), which permits unrestricted use, distribution, and reproduction in any medium, provided you give appropriate credit to the original author(s) and the source, provide a link to the Creative Commons license, and indicate if changes were made. The Creative Commons Public Domain Dedication waiver (http:// creativecommons.org/publicdomain/zero/1.0/) applies to the data made available in this article, unless otherwise stated. up to 10 billion dollars per year only in the United States $[2,5]$. Most patients affected by GERD experience resolution of symptoms on administration of proton-pump inhibitors (PPIs) [6], but these drugs do not seem to alleviate symptoms nor protect the esophageal mucosa in case of erosive lesions [2]. Based on the findings of conventional endoscopy and histopathological examination, GERD is categorized into three progressive stages: non-erosive reflux disease, reflux esophagitis, and Barrett's esophagus [7,8]. As mucosal integrity is a pivotal factor in preventing inflammatory and erosive forms [9], new therapeutic approaches are focusing on drugs capable of being retained within the esophagus, thus coating and protecting its mucosal layer without inducing esophageal blockage [10]. Nowadays, a variety of formulas based on either hyaluronic acid and chondroitin sulfate [11] or sodium alginate [12] is available. When it comes to diagnosis of this pathology, different methods, such as PPI trials and endoscopic evaluation, can be used [13]. Nonetheless, it was recently proved how the most reliable diagnostic test is $24 \mathrm{~h} \mathrm{pH}$ monitoring [14], as it allows for the quantification of acid exposure and 
correlation between symptoms and reflux episodes [2]. Moreover, as the type of the reflux can differ (acid, non-acid, liquid, or gas) depending on the variability of the gastric contents composition [15], $\mathrm{pH}$ monitoring can be combined with multichannel intraluminal impedance and $\mathrm{pH}(\mathrm{MII}-\mathrm{pH})$ measurement to gain more info regarding the nature of the reflux itself [16]. Indeed, MII was first introduced to investigate the characteristics of the reflux in the esophagus [17] and can be used both as a diagnostic tool [15] and therapy outcome monitoring [9], and has also been proven to be indicative of mucosal lesions [18].

Many ex vivo models have been validated and optimized to investigate the esophageal coating potential of different medical devices [19-21], always using mucosal portions of porcine esophagi. Overall, porcine esophagi represent a good model to perform screening tests, as they can be easily collected at slaughterhouses and present more histological and morphological similarities with the human esophagus if compared to rodents [22]. While ex vivo models have been used to test the efficacy of medical devices through histological and $\mathrm{pH}$ analyses [11], the measurement of impedance in an ex vivo model is still lacking and may provide important new insight into experimental trials.

Therefore, the aim of this study was to set up an ex vivo porcine model of acid-induced damage and to evaluate its translatability and reproducibility by means of MII, pH, histology, and Evans blue (EB) permeability assay.

\section{Materials and Methods}

\section{Ethical approval}

The present work included tissues collected at the slaughterhouse from carcasses destined for human consumption, thus replacing killing animals for tissue sampling in accordance with the 3Rs; no animal was sacrificed solely for the purpose of this experiments therefore, no ethical approval was needed.

\section{Study period and location}

The experiments were carried out from July 2017 to April 2018 at the Physiology Laboratories of the Department of Veterinary Medical Sciences of the University of Bologna (Ozzano dell'Emilia, Italy).

\section{Organs collection and preparation}

Thirteen $(n=13)$ swine esophagi were collected at a local slaughterhouse from commercially available European breed pigs and transferred within $2 \mathrm{~h}$ in cooled saline to the physiology labs of the Department of Veterinary Medical Sciences of the University of Bologna. On arrival, each esophagus was thoroughly rinsed with tap water (both inside and outside) and ablated of the cranial and caudal sphincters, maintaining a total length of $30 \mathrm{~cm}$ (Figure-1A). Only organs without visible lesions were used for experimental purposes. Organs were then pinned upright (cranial end at the top and caudal end at the bottom) on polystyrene support and placed, with an inclination of approximately $45^{\circ}$, in a thermostatic hood (Climatic Hood 810; ASAL s.r.l, Cernusco sul Naviglio, Italy) set at $37^{\circ} \mathrm{C}$ (Figure-1B), as previously described [11]. Organs were externally kept moist by means of wet tissue paper. Once positioned, two infusion tubes connected to dedicated pumps were inserted in the top end of the organ for the infusion of the different solutions. Finally, anion-sensitive field-effect transistor (ISFET) MII-pH probe (pHTip 1pH 8E, UNISENSOR AG, Attikon, Switzerland) was inserted in each esophagus for a total length of $25 \mathrm{~cm}$; adherence to the mucosa was insured by applying a delicate pressure on the organs. Three esophagi, used as controls for histology, were only set up and left in the thermostatic hood for $3.5 \mathrm{~h}$ (overall time of the experimental protocol), before sampling.

\section{Solutions}

To perform basal "wet" recordings, similar to water deglutition, commercially available sterile saline was used $(\mathrm{NaCl} 0.9 \%$; S.A.L.F. Spa Laboratorio Farmaceutico, Cenate Sotto, Italy), while to mimic

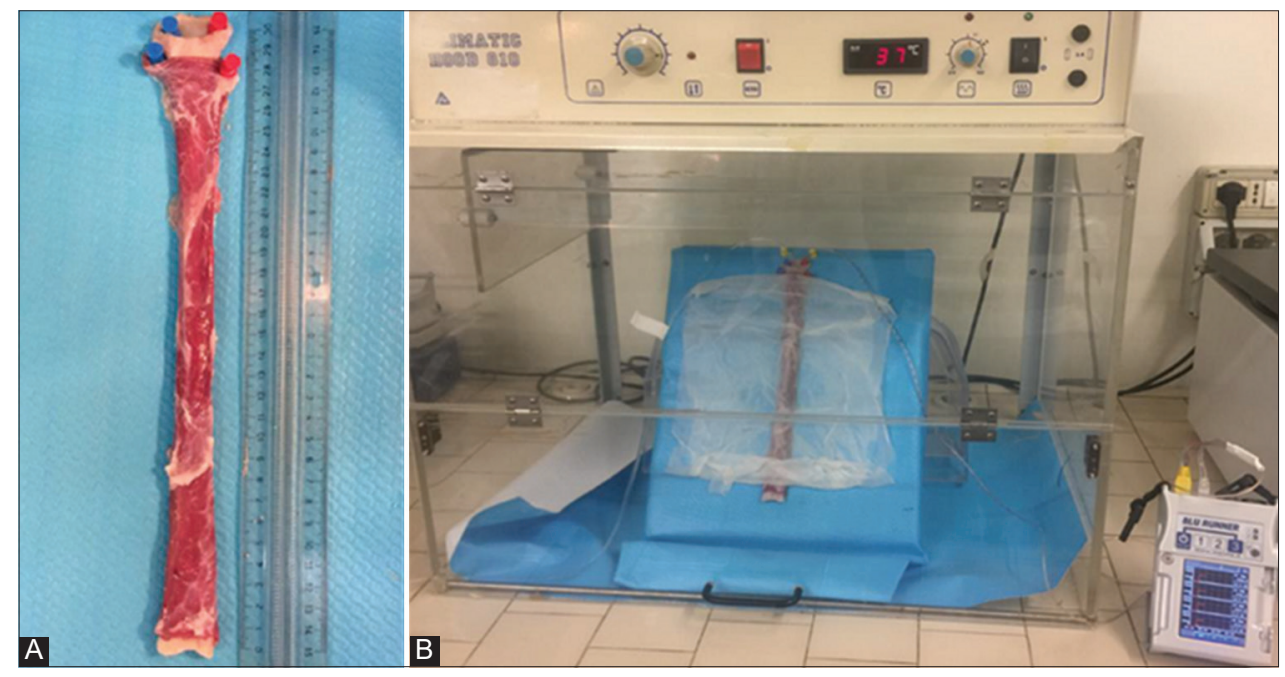

Figure-1: (A) Esophagus ablated of the upper and lower sphincters, cut to a final length of $30 \mathrm{~cm}$ and pinned to the support. (B) Experimental set up in the thermostatic hood. 
acid gastroesophageal reflux, a $0.1 \mathrm{M}$ hydrochloric acid solution was freshly prepared on the day of each trial (hydrochloric acid 37 \% RPE, CARLO ERBA Reagents s.r.l, Cornaredo, Italy). Artificial saliva, used to simulate the physiological environment of the esophagus, was prepared as previously described [10]. All the solutions were used at room temperature.

\section{Experimental protocol}

The experimental protocol, applied to 10 esophagi, is reported in Table-1. For the first $15 \mathrm{~min}$, no infusion was performed to analyze baseline mucosal impedance and $\mathrm{pH}$. Then, saline washing was started with an infusion rate of $600 \mathrm{~mL} / \mathrm{h}$ and lasted for $15 \mathrm{~min}$ followed by $30 \mathrm{~min}$ of "dry" registration. Afterward, to induce the acid damage, acid solution $(\mathrm{HCl} 0.1 \mathrm{M})$ infusion was performed for $30 \mathrm{~min}$ at the same infusion rate followed by a final registration of $120 \mathrm{~min}$. During the final period of registration, two saliva washings $(10 \mathrm{~mL}$ infused in $1 \mathrm{~min}$, each) were done at 45 and $90 \mathrm{~min}$ after the end of the acid treatment. For each infusion (saline, acid, and saliva), fluids were collected in a Petri dish placed under the organs and measured. The total duration of the experimental protocol was $3.5 \mathrm{~h}$.

\section{MII-pH}

MII-pH was recorded for the entire duration of the protocol using a modified version of a commercial impedance recorder (BLU RUNNER; Menfis Biomedical s.r.l., Bologna, Italy). The disposable ISFET probes, with 1 distal $\mathrm{pH}$ sensor and 6 recording impedance channels, were calibrated $(1$ and $7 \mathrm{pH}$ standard buffers, BIOTECH, Vesoul, France) and activated with tap water according to the manufacturer's instruction before each use. At the end of each experiment, data regarding $\mathrm{pH}$ and impedance were exported using the software IMP/HS Dyno 3000. Five different time points were used for the inferential statistical analysis as shown in Table-2: The end of the baseline recording $(\mathrm{t} 0)$, the end of the saline solution infusion $(\mathrm{t} 1)$, the end of the post-saline infusion recording ( $\mathrm{t} 2)$, the end of the acid infusion (t3), and, finally, $30 \mathrm{~min}$ of post-acid infusion recording ( $t 4)$.

To evaluate the influence of the two different infusions (saline and acid solution), percentage of decreases was calculated using the end of the baseline recording (t0) as $100 \%$, according to the following formulas:

$\mathrm{NaCl}$ infusion $=\mathrm{x}: 100=(\mathrm{t} 1-\mathrm{t} 0): \mathrm{t} 0$

$\mathrm{HCl}$ infusion $=\mathrm{x}: 100=(\mathrm{t} 3-\mathrm{t} 0): \mathrm{t} 0$

On the other hand, to evaluate the MII-pH recovery $30 \mathrm{~min}$ after the end of each infusion, percentages were calculated using the difference between the recordings at the end of the infusions and the baseline as $100 \%$, according to the following formulas:

$\mathrm{NaCl}$ infusion $=\mathrm{x}: 100=(\mathrm{t} 2-\mathrm{t} 1):(\mathrm{t} 0-\mathrm{t} 1)$

$\mathrm{HCl}$ infusion $=\mathrm{x}: 100=(\mathrm{t} 5-\mathrm{t} 3):(\mathrm{t} 0-\mathrm{t} 3)$

\section{Sampling for EB permeability assay and histology}

At the end of the experimental protocol, each esophagus, including the three control ones, was longitudinally cut before ISFET probe removal and the exposed
Table-1: Experimental protocol.

\begin{tabular}{ll}
\hline Time (min) & Procedures \\
\hline $0-15$ & Baseline recording \\
$15-30$ & Saline infusion $600 \mathrm{~mL} / \mathrm{h}$ \\
$30-60$ & Post-saline recording \\
$60-90$ & Acid solution infusion $600 \mathrm{~mL} / \mathrm{h}$ \\
$90-210$ & Post-acid recording \\
135 & $1^{\text {st }}$ saliva bolus \\
180 & $2^{\text {nd }}$ saliva bolus \\
\hline
\end{tabular}

Table-2: Time points for MII-pH analyses.

\begin{tabular}{llc}
\hline $\begin{array}{l}\text { Time } \\
\text { point }\end{array}$ & Event & $\begin{array}{c}\text { Minute of } \\
\text { recording }\end{array}$ \\
\hline t0 & End of baseline recording & $15 \mathrm{~min}$ \\
t1 & End of saline infusion & $30 \mathrm{~min}$ \\
t2 & 30 min of post-saline infusion recording & $60 \mathrm{~min}$ \\
t3 & End of acid infusion & $90 \mathrm{~min}$ \\
t4 & 15 min of post-acid infusion recording & $105 \mathrm{~min}$ \\
t5 & 30 min of post-acid infusion recording & $120 \mathrm{~min}$ \\
t6 & Before the first saliva bolus & $135 \mathrm{~min}$ \\
t7 & Before the second saliva bolus & $180 \mathrm{~min}$ \\
t8 & End of recording & $210 \mathrm{~min}$ \\
\hline
\end{tabular}

MII-pH: Multichannel intraluminal impedance and $\mathrm{pH}$

mucosa was carefully washed for $5 \mathrm{~min}$ in saline solution ( $\mathrm{NaCl} 0.9 \%$ ) for macroscopic observations. A full-thickness biopsy was performed $7 \mathrm{~cm}$ above the last impedance recording channel and divided into two pieces: One for EB permeability assay and one for histology.

\section{EB permeability assay}

For the EB permeability assay, $500 \mu \mathrm{L}$ of freshly prepared EB solution $(10 \mathrm{mg} / \mathrm{mL}$ in $\mathrm{NaCl} 0.9 \%$ saline solution; Sigma-Aldrich, NJ, USA) were placed on top of each mucosa fragment, previously placed upward in a Petri dish. After $10 \mathrm{~min}$, samples were washed twice in saline solution $(\mathrm{NaCl} 0.9 \%)$ : The first wash consisted of two rapid dips in $100 \mathrm{~mL}$ of saline solution, while the second one, in $50 \mathrm{~mL}$ of saline, lasted $5 \mathrm{~min}$. At the end, all samples were trimmed and divided into two aliquots to get technical duplicates. Aliquots were then dried at $37^{\circ} \mathrm{C}$ in a thermostatic hood for $30 \mathrm{~min}$ and the mucosal layer was isolated, immediately weighed, placed in $3 \mathrm{~mL}$ of formamide (Sigma-Aldrich, NJ, USA) and incubated for $48 \mathrm{~h}$ at $50^{\circ} \mathrm{C}$ to extract $\mathrm{EB}$ dye. Colorimetric measurements were performed using a spectrophotometer (Gene Quant 1300; GE Healthcare, UK) at the maximum absorption for EB $(620 \mathrm{~nm})$. Micrograms of EB per mg of tissue were quantified using a standard curve (0.025$25 \mu \mathrm{g} / \mathrm{mL}$ EB in formamide) and samples' weights. Data of the two technical replicates were averaged.

\section{Histology}

Samples for histology were fixed overnight in cold $4 \%$ formalin solution (in $0.1 \mathrm{M}$ phosphate buffer, $\mathrm{pH} 7.4$, Kaltek, Italy) and then moved into $25 \%$ sucrose (Sigma-Aldrich, NJ, USA) solution in phosphate buffer at $4^{\circ} \mathrm{C}$ for at least $24 \mathrm{~h}$. Afterward, samples were embedded in OCT (Sakura Finetek, USA). Ten micrometer sections were cut at a Leica CM1950 cryostat (Leica, Wetzlar, Germany), mounted on microscope's 
slides, and stained with hematoxylin and eosin (H\&E) according to standard procedure. Images were obtained using a camera (Digital C-Mount Camera, Nikon, Tokyo, Japan) installed on an inverted microscope (ECLIPSE TS100, Nikon, Tokyo, Japan) using the Alexasoft X-Elit image analysis system (Alexasoft, Florence, Italy). For each slide, six measures were done to evaluate the thickness of the mucosal layer.

\section{Statistical analysis}

Statistical analysis was performed using the software GraphPad Prism (GraphPad, La Jolla, CA, USA). The distribution of all data was assessed by means of Shapiro-Wilk test. Regarding MII-pH, to compare the percentages of decrease and increase between saline and acid infusions in the experimental esophagi $(n=10)$, either paired t-test or Wilcoxon test was used. To evaluate differences between the baseline values $(\mathrm{t} 0)$ and the different time points during the post-acid infusion recordings (from t3 to t8), a repeated measure (RM) ANOVA, followed by Tukey's post hoc test, was performed. The results of EB permeability assay for control esophagi and acid-damaged ones were compared by means of Welch's $t$-test, while for histological data, Mann-Whitney's $U$-test was applied. Significance was set for $\mathrm{p}<0.05$ (Confidence Interval 95\%).

\section{Results}

All the used esophagi did not show any macroscopically visible alteration, on evaluation of the mucosa after longitudinal cutting, imputable to both pre-existing condition and the experimental protocol itself. Raw data of the different esophagi were averaged. At the end of each infusion, the entire volume of the used solution was recovered, yet with small temporal differences: Saline and acid solutions were completely recovered within 15 $\mathrm{s}$ from the end of infusion, while saliva within $40 \mathrm{~s}$.

\section{MII-pH}

For each esophagus, the impedance recordings of the six channels of the ISFET probe were averaged. The effects of the infusion of saline $(\mathrm{NaCl} 0.9 \% ; \mathrm{t} 1)$ and acid $(\mathrm{HCl} 0.1 \mathrm{M}$; $\mathrm{t} 3)$ solutions on MII-pH are represented in Figure- 2 as percentage of decrease in comparison to the baseline values ( $\mathrm{t} 0$ ). The decrease in both parameters induced by the acid solution was statistically more marked when compared to the one induced by saline solution.

The percentages of MII-pH recovery $30 \mathrm{~min}$ after the end of each infusion $(\mathrm{NaCl} 0.9 \% \mathrm{t} 2 ; \mathrm{HCl} 0.1$ $\mathrm{M} \mathrm{t5)}$ are represented in Figure-3. Also, in this case, both parameters show a statistically significant difference when comparing the two solutions, with better recovery recorded after the saline infusion.

The results of the RM ANOVA for the evaluation of absolute MII-pH values at different time points after the acid solution $(\mathrm{HCl} 0.1 \mathrm{M})$ infusions are represented in Figure-4. When compared to the baseline (t0), MII-pH values at the end of the infusion ( $\mathrm{t} 3$ ) were statistically lower. After 15 min of post-infusion

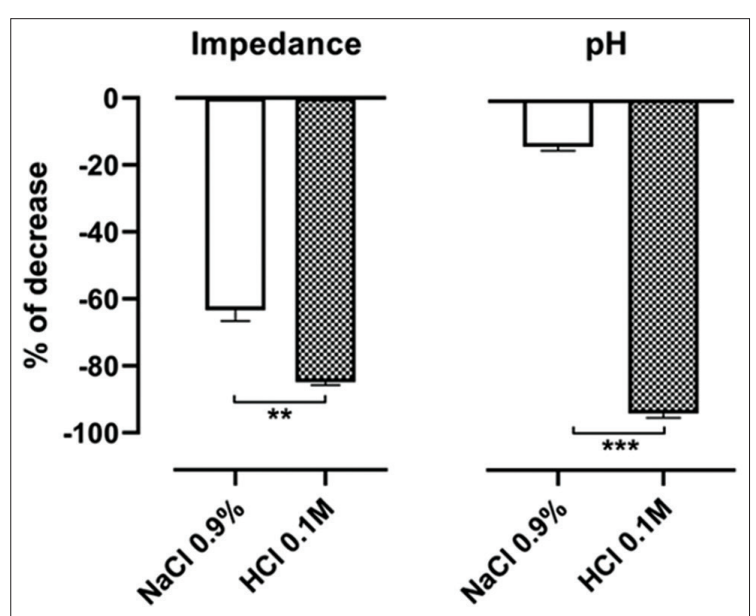

Figure-2: Percentages of decrease in MII and $\mathrm{pH}$ after saline $(\mathrm{NaCl} 0.9 \%)$ and acid $(\mathrm{HCl} 0.1 \mathrm{M})$ infusions in comparison to the baseline values. Data are reported as means and standard error of the mean. (Impedance: Paired $t$-test; $\mathrm{pH}$ : Wilcoxon test; $* * \mathrm{p}<0.01 ; * * * \mathrm{p}<0.001)$.

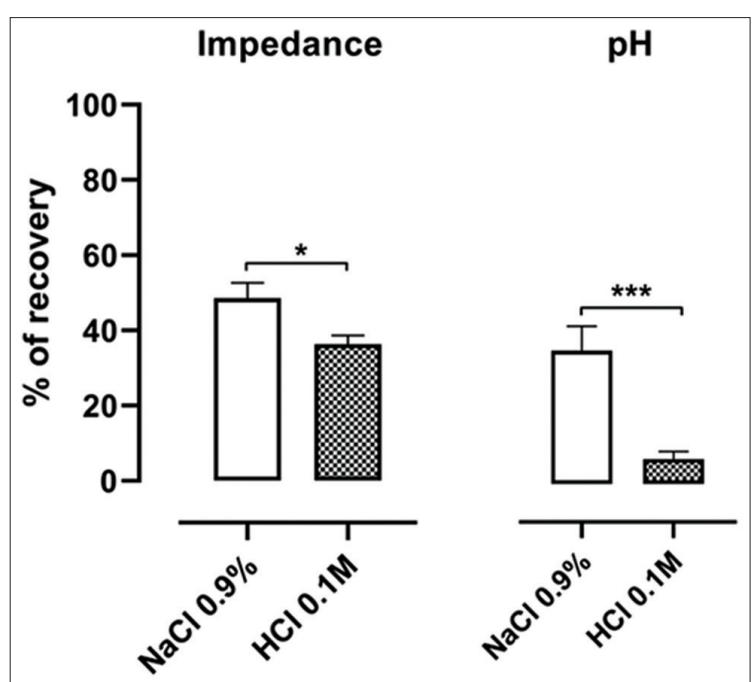

Figure-3: Percentages of recovery in multichannel intraluminal impedance and $\mathrm{pH} 30 \mathrm{~min}$ after saline $(\mathrm{NaCl}$ $0.9 \%)$ and acid $(\mathrm{HCl} 0.1 \mathrm{M})$ infusions. Data are reported as means and standard error of the mean (paired $t$-test; $* \mathrm{p}<0.05 ; * * * \mathrm{p}<0.001)$.

recording (t4), MII significantly increases and remains constant without ever reaching the baseline values. On the other hand, $\mathrm{pH}$ values remain low and only increase after the second saliva bolus ( $\mathrm{t} 7$ ), reaching statistically similar values when compared to the baseline (t0).

\section{EB permeability assay}

The results of the comparison of the EB permeability assay performed on control $(n=3)$ and experimental $(\mathrm{n}=10)$ esophagi are represented in Figure-5. A statistically significant increase was recorded for the samples obtained by the esophagi that underwent the experimental protocol and were, therefore, exposed to the acid solution $(\mathrm{HCl} 0.1 \mathrm{M})$.

\section{Histology}

The results of the histological analysis of the mucosal thickness on control $(\mathrm{A} ; \mathrm{n}=3)$ and experimental $(B ; n=10)$ esophagi and their comparison are 


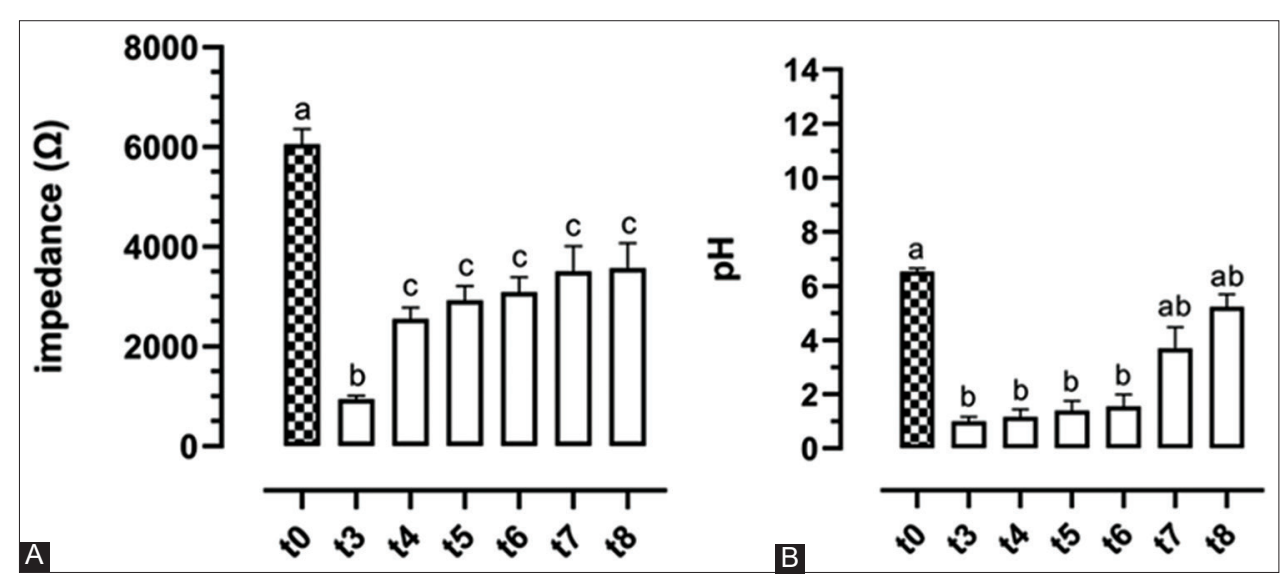

Figure-4: Multichannel intraluminal impedance $(\mathrm{A})$ and $\mathrm{pH}(\mathrm{B})$ values at different time points after acid solution $(\mathrm{HCl} 0.1 \mathrm{M})$ infusion in comparison to the baseline (t0). Different letters indicate statistically significant differences between time points (RM ANOVA, Tukey's post hoc test; $\mathrm{p}<0.05$ ). $\mathrm{t} 3=$ end of acid infusion; $\mathrm{t} 4=15$ min of post-acid infusion recording; $\mathrm{t} 5=30 \mathrm{~min}$ of post-acid infusion recording; $\mathrm{t} 6=$ =before the first saliva bolus; $\mathrm{t} 7=$ before the second saliva bolus; $\mathrm{t} 8=\mathrm{end}$ of recording.

represented in Figure-6. As for the EB, a statistically significant increase in mucosal thickness was recorded for the samples obtained by the esophagi that underwent the experimental protocol and were, therefore, exposed to the acid solution ( $\mathrm{HCl} 0.1 \mathrm{M})$.

\section{Discussion}

As previously stated, the aim of the work was to create an ex vivo model capable of mimicking esophageal acid reflux for preliminary screening of medical devices and therapies. It is indeed extremely important to scrutinize and develop preclinical models to reduce the number of animals enrolled in in vivo studies. In such scenario, the possibility to use organs collected at slaughterhouses represents a good option in full respect of the 3 Rs principle $[23,24]$ and has already been described for the porcine species $[11,25]$. Nonetheless, such collection procedure has some limitations including the lack of sterility and the necessity for trained personnel capable of noticing potential lesions caused by slaughter itself.

All of the esophagi used for the experimental protocol showed basal levels of MII-pH similar to the ones recorded in healthy adult humans in physiological conditions [26]. This finding is important as it validates the already known anatomical/functional similarities of pigs when compared to humans. Indeed, in the past decades, this species has emerged in the field of translational medicine, especially when it comes to gastroenterology. This is potentially related to the fact that, out of the most commonly used large animal models, pigs are non-ruminant omnivorous and show metabolic patterns close to humans.

The same model was already proposed and used to evaluate anti-GERD devices by the same research group [11], but this work shows the addition of a better definition of the damage by means of a quantitative evaluation of EB permeability assay and of a well-recognized method in human gastroenterology as MII-pH analysis. In physiological conditions, when

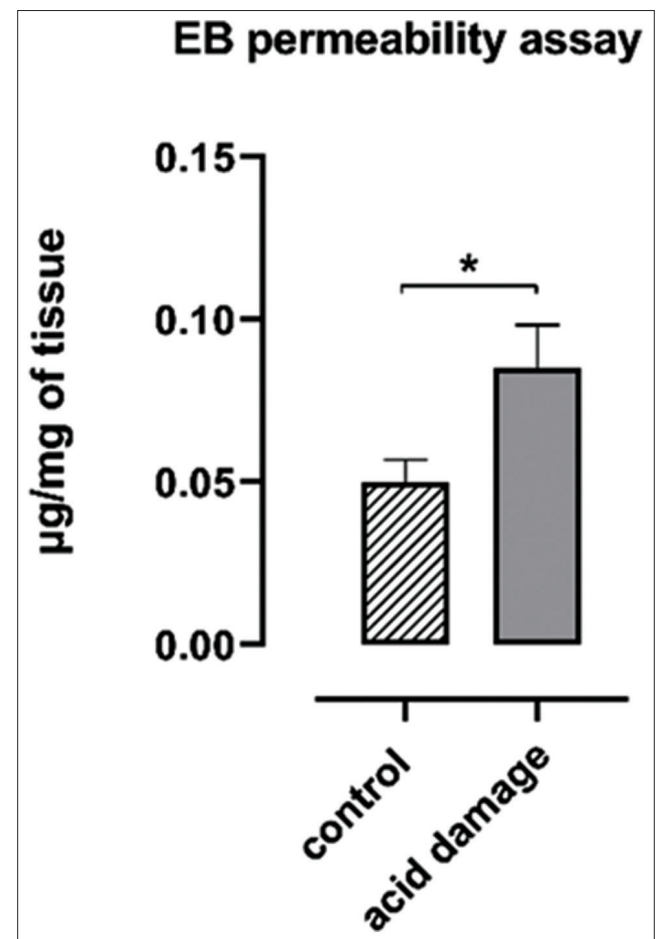

Figure-5: Evans blue permeability assay on control $(n=3)$ and experimental $(n=10)$ esophagi. Data are reported as means and standard error of the mean (Welch's $t$-test, $* p<0.05)$.

the mucosal layers are intact, electrical impedance is high and only drops when liquids wet the above-mentioned layers. On the other hand, in case of anatomical alterations of the mucosa such as erosive lesions, the baseline impedance is lower $[27,28]$. When it comes to GERD, MII-pH is used to identify and classify reflux episodes: A reflux episode is defined as a fall in impedance of $\leq 50 \%$ of baseline and, when $\mathrm{pH}$ lowers and remains $\leq 4$ for at least $5 \mathrm{~s}$, can be considered acid [29]. According to the results, it can be stated that the infusion of acid solution, $\mathrm{HCl} 0.1 \mathrm{M}$ in this peculiar case, has indeed determined the simulation of an acid reflux event. 


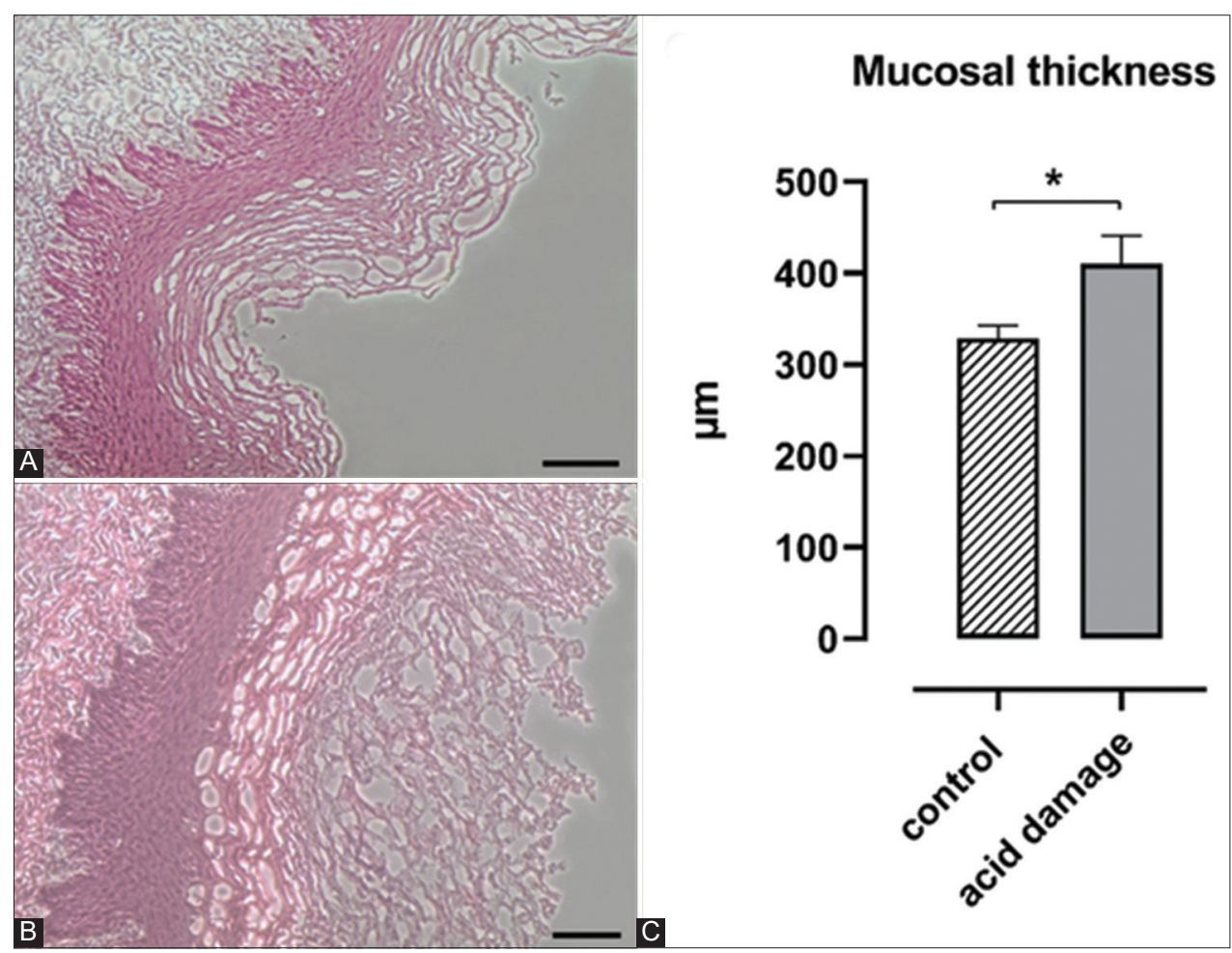

Figure-6: Hematoxylin-eosin staining of control (A) and experimental (B) esophagi (100x; bars: $100 \mu \mathrm{m})$. Comparison of mucosal thickness between the two groups (C). Data are reported as mean and standard error of the mean (Mann-Whitney $U$-test, $* p<0.05)$.

One of the downsides of the proposed experimental model is the lack of the physiological peristaltic movements characteristic of the esophageal functionality. Unfortunately, such statement can be applied to the majority of the currently available ex vivo models, and work needs to be done to implement this gap potentially by means of mechanical devices. Nonetheless, the capability to recover the entire volume of the infused solutions from the lower end of the organs indicates a correct flow within the esophagi and the absence of liquids accumulation, also confirmed by MII-pH. The temporal delay observed for the recovery of the saliva when compared to the other solutions has to be imputed to the viscosity of the artificial saliva and further supports the sensitivity of the model.

The impedance results show how it dropped, as expected, during every infusion, proving not only the sensitivity of the recording itself but also most important that the probe was always in contact with the mucosal layer. The latter is pivotal for a correct recording and having the chance to visualize the recording "live" is pivotal in such trials. Nonetheless, not all the commercially available impedance recorders offer this feature, mainly to preserve the battery's autonomy during $12-24 \mathrm{~h}$ recording. For this particular trial, a modified impedance recorder was developed to allow a battery autonomy of at least $4 \mathrm{~h}$ with active screen. The sensitivity of the method is further confirmed by the fact that the decrease in MII determined by the acid solution was statistically more severe, indicating that what is registered cannot only be imputed by the wetting of the mucosal layer but also by the nature of the used solution.

After the two impedance drops determined by saline and acid solution infusion, the patterns of recovery were statistically different, with a faster and more marked recovery after the infusion of saline. This result seems to confirm the hypothesis of acid-induced damage to the mucosa, that is, not capable of completely restoring the normal baseline electric impedance. To further support such statement, the results of the RM ANOVA analysis (Figure-4A) show how MII increases within the first $15 \mathrm{~min}$ after the acid infusion, but immediately reaches a plateau thereafter (from $t 4$ to the end of the recording) and never gets back to normal values despite the two saliva boluses.

All the discussions regarding MII are strengthened by the results of $\mathrm{pH}$ (Figure-4B), recorded by the same probe (MII-pH) and the same recorder. Being the $\mathrm{pH}$ of the used solution standardized, the results are coherent with the anticipated outcome. Indeed, the saline solution $(\mathrm{NaCl} 0.9 \%)$ always induced statistically milder $\mathrm{pH}$ variations when compared to the ones induced by the acid solution. Nonetheless, as opposite to MII, the $\mathrm{pH}$ showed a better recovery during the $2 \mathrm{~h}$ of post-acid infusion recording, reaching levels comparable to the baseline at the last 2 time points. Such different behavior is imputable by the nature of the parameter itself, directly correlated to the used solutions rather than the integrity of the mucosa. In this case, the saliva boluses may have better contributed to the recovery as opposed to what happened for MII. 
The results of EB permeability assay and histology confirm what recorded by MII and demonstrate the hypothesized acid-induced damage to the mucosa. EB is known to bind quantitatively to albumin in vivo and in vitro and so it could be used as an indicator of esophageal mucosa permeability [30-32]. A previous experiment with the same model showed that the absorption of EB was due to the increased mucosal permeability caused by the acid perfusion since the intact mucosa did not absorb the dye [11]. To obtain a more objective measurement of the absorption, unlike the previous experiment, we used a method of extraction and quantification of the dye instead of the visual analysis of the staining. The comparison between the 10 experimental esophagi and the 3 control ones highlighted how the acid solution infusion allowed higher amounts of EB to permeate into the sample. In physiological conditions, an intact mucosal layer should almost completely prevent stains like EB to penetrate the deeper tissue layers, as seen in the control organs. The higher concentrations of EB found in acid-treated esophagi are, therefore, indicative of increased mucosal permeability, again already suggested by the other results previously discussed. Such hypothesis was definitively confirmed by histology that showed an increase in mucosal thickness, due to exfoliation and loss of continuity.

\section{Conclusion}

According to the results of the present study, the hereby proposed model of esophageal acid-induced damage seems to be repeatable, reliable, and achievable using organs collected at the slaughterhouse in respect of the 3 Rs principles. MII recording, largely used in clinical settings, proved to have good sensitivity in detecting mucosal alterations also in ex vivo trials. Overall, the model may be suitable for preliminary screening of both drugs and medicals devices developed to treat GERD, in light of its relatively low ethical and economic impacts.

\section{Authors' Contributions}

MLB, MF, and FB conceived and designed the study. DV, AE, RS, and GC performed the experiments, and AE analyzed the data. DV, RS, and GC drafted the manuscript. All authors revised the final manuscript and approved it.

\section{Acknowledgments}

The authors would like to thank Menfis Biomedical s.r.l. for the collaboration in developing the modified MII-pH recorder. This research was supported by funding from the University of Bologna RFO project (Via Zamboni 33, Bologna, 40126, Italy).

\section{Competing Interests}

The authors declare that they have no competing interests.

\section{Publisher's Note}

Veterinary World remains neutral with regard to jurisdictional claims in published institutional affiliation.

\section{References}

1. Vakil, N., van Zanten, S.V., Kahrilas, P., Dent, J. and Jones, R. (2006) The Montreal definition and classification of gastroesophageal reflux disease: A global evidence-based consensus. Am. J. Gastroenterol., 101(8): 1900-1920.

2. Patti, M.G. (2016) An evidence-based approach to the treatment of gastroesophageal reflux disease. JAMA Surg., 151(1): 73-78.

3. Kahrilas, P.J., Altman, K.W., Chang, A.B., Field, S.K., Harding, S.M., Lane, A.P., Lim, K., McGarvey, L., Smith, J. and Irwin, R.S. (2016) Chronic cough due to gastroesophageal reflux in adults: CHEST guideline and expert panel report. Chest, 150(6): 1341-1360.

4. El-Serag, H.B., Sweet, S., Winchester, C.C. and Dent, J. (2014) Update on the epidemiology of gastro-oesophageal reflux disease: A systematic review. Gut, 63(6): 871-880.

5. Joish, V.N., Donaldson, G., Stockdale, W., Oderda, G.M., Crawley, J., Sasane, R., Joshua-Gotlib, S. and Brixner, D.I. (2005) The economic impact of GERD and PUD: Examination of direct and indirect costs using a large integrated employer claims database. Curr. Med. Res. Opin., 21(4): 535-544.

6. Becher, A. and El-Serag, H. (2011) Systematic review: The association between symptomatic response to proton pump inhibitors and health-related quality of life in patients with gastro-oesophageal reflux disease. Aliment Pharmacol. Ther., 34(6): 618-627.

7. Lv, J., Liu, D., Ma, S.Y. and Zhang, J. (2013) Investigation of relationships among gastroesophageal reflux disease subtypes using narrow band imaging magnifying endoscopy. World J. Gastroenterol., 19(45): 8391-8397.

8. Pace, F. and Bianchi Porro, G. (2004) Gastroesophageal reflux disease: A typical spectrum disease (a new conceptual framework is not needed). Am. J. Gastroenterol., 99(5): 946-949.

9. Woodland, P., Lee, C., Duraysami, Y., Farré, R., Dettmar, P. and Sifrime, D. (2013) Assessment and protection of esophageal mucosal integrity in patients with heartburn without esophagitis. Am. J. Gastroenterol., 108(4): 535-543.

10. Batchelor, H.K., Banning, D., Dettmar, P.W., Hampson, F.C., Jolliffe, I.G. and Craig, D.Q.M. (2002) An in vitro mucosal model for prediction of the bioadhesion of alginate solutions to the oesophagus. Int. J. Pharm., 238(1-2): 123-132.

11. Di Simone, M.P., Baldi, F., Vasina, V., Scorrano, F., Bacci, M.L., Ferrieri, A. and Poggioli, G. (2012) Barrier effect of $\operatorname{Esoxx}\left({ }^{\circledR}\right)$ on esophageal mucosal damage: Experimental study on ex-vivo swine model. Clin. Exp. Gastroenterol., 5: 103-107.

12. Richardson, J.C., Dettmar, P.W., Hampson, F.C. and Melia, C.D. (2005) Oesophageal bioadhesion of sodium alginate suspensions 2. Suspension behaviour on oesophageal mucosa. Eur. J. Pharm. Sci., 24(1): 107-114.

13. Ates, F. and Vaezi, M.F. (2014) New approaches to management of PPI-refractory gastroesophageal reflux disease. Curr. Treat. Options Gastroenterol., 12(1): 18-33.

14. Patti, M.G., Diener, U., Tamburini, A., Molena, D. and Way, L.W. (2001) Role of esophageal function tests in diagnosis of gastroesophageal reflux disease. Dig. Dis. Sci., 46(3): 597-602.

15. Sifrim, D., Silny, J., Holloway, R.H. and Janssens, J.J. (1999) Patterns of gas and liquid reflux during transient lower oesophageal sphincter relaxation: A study using intraluminal electrical impedance. Gut, 44(1): 47-54.

16. Vela, M.F. (2009) Non-acid reflux: Detection by multichannel intraluminal impedance and $\mathrm{pH}$, clinical significance 
and management. Am. J. Gastroenterol., 104(2): 277-280.

17. Frieling, T., Hermann, S., Kuhlbusch, R., Enck, P., Silny, J., Lübke, H.J., Strohmeyer, G. and Haeussinger, D. (1996) Comparison between intraluminal multiple electric impedance measurement and manometry in the human oesophagus. Neurogastroenterol. Motil., 8(1): 45-50.

18. Hobbs, P. and Gyawali, C.P. (2018) The role of esophageal $\mathrm{pH}$-impedance testing in clinical practice. Curr. Opin. Gastroenterol., 34(4): 249-257.

19. Krüger, L., Pridgen, T.A., Taylor, E.R., Garman, K.S. and Blikslager, A.T. (2020) Lubiprostone protects esophageal mucosa from acid injury in porcine esophagus. Am. J. Physiol. Gastrointest. Liver Physiol., 318(4): G613-G623.

20. Smart, J.D., Dunkley, S., Tsibouklis, J. and Young, S. (2015) An evaluation of the adhesion of solid oral dosage form coatings to the oesophagus. Int. J. Pharm., 496(2): 299-303.

21. Smart, J.D., Dunkley, S., Tsibouklis, J. and Young, S. (2013) An in vitro model for the evaluation of the adhesion of solid oral dosage forms to the oesophagus. Int. J. Pharm., 447(12): 199-203

22. Krüger, L., Gonzalez, L.M., Pridgen, T.A., McCall, S.J., von Furstenberg, R.J., Harnden, I., Carnighan, G.E., Cox, A.M., Blikslager, A.T. and Garman, K.S. (2017) Ductular and proliferative response of esophageal submucosal glands in a porcine model of esophageal injury and repair. Am. J. Physiol. Gastrointest. Liver Physiol., 313(3): G180-G191.

23. Russell, W.M.S. and Burch, R.L. (1959) The Principles of Humane Experimental Technique. Johns Hopkins Bloomberg School of Public Health, Methuen, London.

24. Tannenbaum, J. and Bennett, B.T. (2015) Russell and Burch's 3Rs then and now: The need for clarity in definition and purpose. J. Am. Assoc. Lab. Anim. Sci., 54(2): 120-132.

25. Bertocchi, M., Rigillo, A., Elmi, A., Ventrella, D., Aniballi, C., Gerardi Scorpio, D., Scozzoli, M., Bettini, G., Forni, M. and Bacci, M.L. (2020) Preliminary assessment of the mucosal toxicity of tea tree (Melaleuca alternifolia) and rosemary (Rosmarinus officinalis) essential oils on novel porcine uterus models. Int. J. Mol. Sci., 21(9): 3350.

26. Weijenborg, P.W., Rohof, W.O.A., Akkermans, L.M.A., Verheij, J., Smout, A.J.P.M. and Bredenoord, A.J. (2013) Electrical tissue impedance spectroscopy: A novel device to measure esophageal mucosal integrity changes during endoscopy. Neurogastroenterol. Motil., 25(7): 574-578, e457-458.

27. Matsumura, T., Arai, M., Ishigami, H., Fujie, M., Ishikawa, K. Akizue, N., Taida, T., Ohta, Y., Hamanaka, S., Okimoto, K., Saito, K., Maruoka, D., Nakagawa, T. and Kato, N. (2018) Evaluation of esophageal mucosal integrity in patients with gastroesophageal reflux disease. Digestion, 97(1): 31-37.

28. Barrett, C., Choksi, Y. and Vaezi, M.F. (2018) Mucosal impedance: A new approach to diagnosing gastroesophageal reflux disease and eosinophilic esophagitis. Curr. Gastroenterol. Rep., 20(7): 33.

29. Mousa, H.M., Rosen, R., Woodley, F.W., Orsi, M., Armas, D. Faure, C., Fortunato, J., O'connor, J., Skaggs, B. and Nurko, S. (2011) Esophageal impedance monitoring for gastroesophageal reflux. J. Pediatr. Gastroenterol. Nutr., 52(2): 129-139.

30. Saria, A. and Lundberg, J.M. (1983) Evans blue fluorescence: Quantitative and morphological evaluation of vascular permeability in animal tissues. J. Neurosci. Methods, 8(1): 41-49.

31. Saria, A. and Lundberg, J.M. (1983) Capsaicin pretreatment inhibits heat-induced oedema in the rat skin. Naunyn Schmiedebergs Arch. Pharmacol., 323(4): 341-342.

32. Zhiyuan, Q., Qingyong, L., Shengming, H. and Hui, M. (2016) Protective effect of rhEPO on tight junctions of cerebral microvascular endothelial cells early following traumatic brain injury in rats. Brain Inj., 30(4): 462-467. 